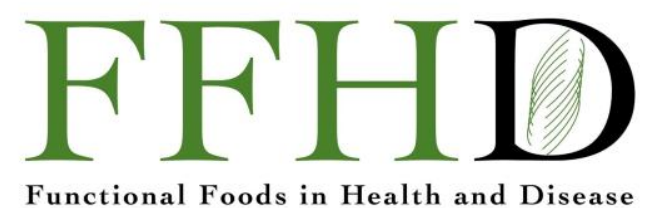

\title{
Frequently used medicinal herbs and spices in weight management: a review
}

\author{
Vered Kaufman-Shruqui, Sheri Sherf-Dagan, \\ Hagit Salem, Daniela Abigail Navarro, Mona Boaz*
}

Department of Nutrition Sciences, Ariel University, Kiryat H'amada St. 3, Ariel, Israel 4070000

*Corresponding Author: Prof. Mona Boaz, Department Chair, Department of Nutrition Sciences, Ariel University, Kiryat H'amada St.3, Ariel, Israel 4070000

Submission Date: July 6 ${ }^{\text {th }}, 2020$; Acceptance Date: July 19 ${ }^{\text {th }}, 2020$; Publication Date: July $23^{\text {rd }}, 2020$

Please cite this article as: Kaufman-Shruqui V., Sherf-Dagan S., Salem H., Navarro D.A., Boaz M. Frequently Used Medicinal Herbs and Spices in Weight Management: A Review. Functional Foods in Health and Disease 2020; 10(7): 305-323 DOI: https://doi.org/10.31989/ffhd.v10i7.719

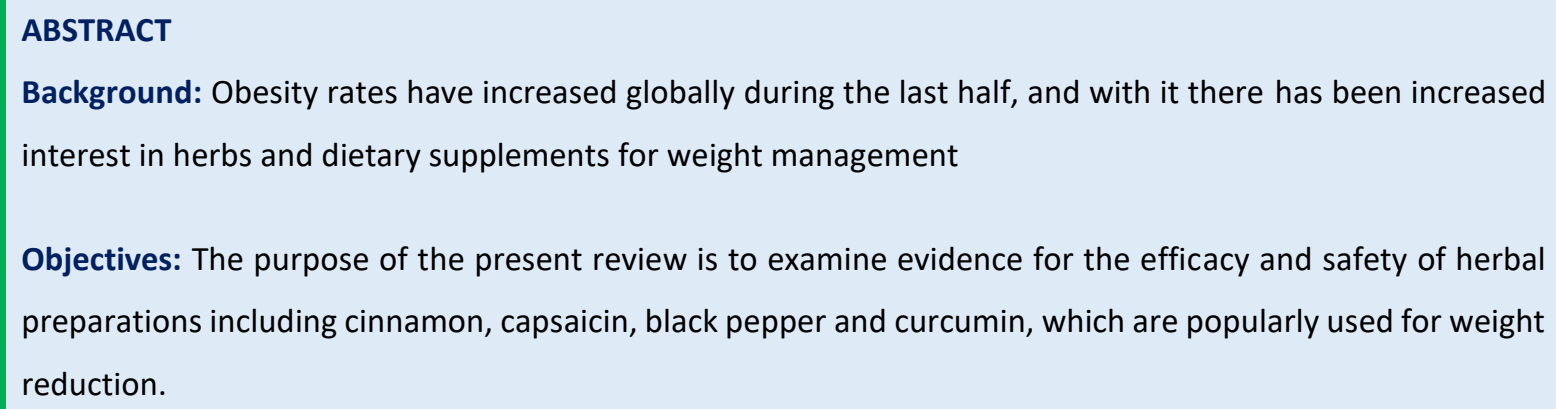

Background: Obesity rates have increased globally during the last half, and with it there has been increased interest in herbs and dietary supplements for weight management

Objectives: The purpose of the present review is to examine evidence for the efficacy and safety of herbal preparations including cinnamon, capsaicin, black pepper and curcumin, which are popularly used for weight reduction.

Methods: A list of herbs and dietary supplements frequently used in weight management was developed by reviewing both scientific and popular literature. Included herbs/supplements were evaluated in terms of preclinical and clinical studies.

Results: Included in the review were cinnamon, capsaicin, black pepper and curcumin. For all of the included herbs, proof of concept could be demonstrated. Few well-designed, adequately powered clinical trials were available but those that were suggested efficacy.

Conclusions: While underlying metabolic/physiological mechanisms have been identified in pre-clinical studies, human studies of efficacy are sparse. An indication towards efficacy in humans exists for all of these products; however, treatment guidelines cannot be developed until better quality randomized clinical trials can provide clear findings. 
Keywords: Cinnamon; Capsaicin; Black Pepper; Curcumin; Weight Management

CFFC 2020. This is an Open Access article distributed under the terms of the Creative Commons Attribution 4.0 License (http://creativecommons.org/licenses/by/4.0)

\section{BACKGROUND}

Obesity rates have steadily increased over the last 40 years and are projected to continue to climb over the next 30 years (1). While obesity is positively associated with Gross Domestic Product Per Capita (GDPPC), the increasing rate in obesity prevalence has shown to be inversely associated with this measure, suggesting that most obesity incidence will occur in lower- and middle-income nations rather than higher income nations (2).

Classified by the American Medical Association as a disease (3), obesity is also a risk factor for many noncommunicable diseases including diabetes (4), hypertension, (5) coronary artery disease (6) and certain cancers (7). This lends an urgency to treating this condition.

Lifestyle management of obesity, which is appropriate for all classes of this condition, includes a balanced, calorie restricted diet, increased exercise (particularly aerobic) and cognitive/behavioral therapy (8). Many individuals augment lifestyle interventions with dietary supplements and herbal preparations believed to be efficacious in enhancing weight loss (9). Indeed, the increase in obesity prevalence has been accompanied by an increase in the use of herbal and dietary supplements for weight reduction (10). But among dietary supplements and herbs most prone to causing adverse health outcomes are those intended for weight loss (11), while evidence supporting the efficacy of these preparations for has not been well established. Nevertheless, herbal preparations are often recommended in the popular literature. We focused on the herbs most frequently cited in these articles: cinnamon, capsaicin, black pepper and curcumin. These herbs appeared on several "Best herbs for weight loss" lists in both print and online magazines and newspaper feature articles directed at the general public.

The purpose of the present review is to examine evidence for the efficacy and safety of herbal preparations popularly used for weight reduction.

\section{METHODS}

The present narrative review seeks to evaluate the safety and efficacy of the following herbs popularly conceived of as appropriate for weight reduction: cinnamon, capsaicin, black pepper and curcumin.

\section{Definitions}

Popular literature: print and electronic media intended for the non-medical, nutrition or life science professional. This included magazine and newspaper feature articles in which herbs and weight control were simultaneously discussed.

Popularly used herbs: A review of the popular literature revealed many "best herbs for weight loss" lists. These lists were recorded, and the most frequently repeated herbs were those selected for inclusion in the present review.

Weight loss: The definition of weight loss was not altered from that used by the authors of the reviewed publications. This was necessary since several of the studies reviewed examined 
mechanism, some used animal models and some used human subjects.

\section{Search Engines}

The present review sought to identify the safety and efficacy of popularly used herbs for weight loss. To this end, the following search engines were employed: PubMed; Medline Plus: Herbs and Dietary Supplements; ResearchGate; and EMBASE. Date restrictions were not included, but only English language publications were included. For each herb cell studies, animal models and human subject research were included. Date restrictions were not utilized, but included studies had to be published in the English language.

\section{Cinnamon}

Cinnamomum is a genus that includes approximately 250 species, the most medically and economically important of which are C. verum (also known as C. zeylanicum) (12) and C. cassia (13). In addition to its use as a in food preparation, cinnamon has been used in traditional medicine to treat diarrhea and other gastrointestinal symptoms (14), menstrual cramps (15), rheumatoid arthritis (16) microbial infection (17) and type 2 diabetes (18).

Pharmacologically active components identified in cinnamon include cinnamic acid, a polyphenol that can inhibit protein kinases essential in cell signaling networks (19). Cinnamaldehyde, another active compound in cinnamon, has antimicrobial (20), antiinflammatory and anti-proliferative bioactivity (21). Another important compound in cinnamon is eugenol, which, like cinnamaldehyde, has both antibacterial and anti-proliferative properties, as well as antioxidant and anti-inflammatory activity (22). Coumarin has anti-coagulant, antiproliferative, anti-microbial, anti-inflammatory and antioxidant properties (23). These bioactive properties may modify chronic disease risk (24).

In 2013, the American Medical Association defined obesity as a chronic disease requiring medical intervention (25). Cinnamon and its compounds have been studied as a potential treatment for obesity.

Cell studies: Cinnamon has long been known to potentiate insulin activity, a characteristic originally attributed to chromium content; however, this concept was subsequently discarded when an association between chromium content and insulin activity was not detected (26). On the other hand, aqueous cinnamon fractions have been shown to inhibit protein tyrosine phosphatase 1, which dephosphorylates the insulin receptor kinase. These fractions also activate insulin receptor kinase autophosphorylation (27). In another study, hydroxychalcone from cinnamon was shown to stimulate glucose uptake. The insulin cascade appears to have been activated by the cinnamonderived hydroxychalcone phosphorylation of the insulin receptor (28). Indeed, insulin receptor phosphorlylation is an established mechanism through which glycemic control can be achieved (29). Insulin signaling stimulates glucose uptake in human skeletal muscle; however, obesity is characterized by defects in this mechanism (30). The ability of cinnamon to phosphorylate the insulin receptor could, then, improve insulin resistance and the metabolic milieu associated with obesity.

Another mechanism through which cinnamon may exert anti-obesity action is transient receptor potential ankyrin 1 (TRPA1). TRPA1 has been shown to improve weight control, insulin and glucose homeostasis and thermogenesis (31). In an in vitro incubation model, cinnamon has been shown to upregulate both TRPA1 and insulin 
receptor genes, improving insulin sensitivity while simultaneously reducing ghrelin secretion (32).

Polyphenolic compounds in cinnamon are accredited with inhibiting intestinal lipid absorption (33), lipolysis and lipogenesis (34). Cinnamon has also been shown to increase thermogenesis and inhibit adipocyte differentiation, resulting in reduced adiposity (35).

Animal studies: Animal studies have generally supported the role of cinnamon in improving insulin resistance, diabetes and obesity. For example, cinnamon-derived phytochemicals including proanthocyanidins, have been shown to lower blood glucose levels in streptozotocin-induced diabetic mice (36). Peroxisome proliferatoractivated receptors (PPAR) alpha and gamma were the mechanisms through which cinnamon water extract reduced blood glucose and serum lipids while mitigating insulin resistance and obesityrelated type 2 diabetes in mice (37). Consistent with these findings, insulin-resistant rats fed a highfructose, high-fat diet supplemented with cinnamon powder exhibited improvement of impaired insulin-signaling gene expression (38). Improved insulin metabolism was also observed in metabolically normal male Wistar rats that were fed cinnamon extract (39). In obese, diabetic rats, cinnamon has been shown to significantly reduce body weight and fat mass in addition to blood glucose and leptin levels, while also normalizing the serum lipid profile (40).

A study examined the metabolic influence of cinnamon bark extract or grape pomace on mice that were fed a high fat diet. Both supplements significantly decreased fat mass gain and adipose tissue inflammation (41).

Taken together, the animal models echo the findings of the in vitro studies; namely, that through a variety of mechanisms, cinnamon can improve insulin and glucose homeostasis, reduce adipocyte differentiation and improve thermogenesis, all of which are associated with reduced body weight. Finally, it has been shown to decrease body fatness and its accompanying inflammation.

Human studies: Cinnamon has been studied in human subjects in a number of clinical trials. Mirroring a phase 1 trial, seven healthy young males underwent an oral glucose tolerance test (OGTT) following ingestion of $5 \mathrm{~g}$ placebo, $5 \mathrm{~g}$ cinnamon, and 12 hours post ingestion of $5 \mathrm{~g}$ cinnamon. Area under the curve (AUC) for OGTT was significantly smaller immediately following and 12 hours after cinnamon ingestion compared to the AUC following placebo ingestion (42). Another study involving healthy young adult males randomized participants to $3 \mathrm{~g} /$ day cinnamon or placebo. After two weeks, cinnamon-treated subjects had significantly reduced AUC for OGTT and significantly improved insulin sensitivity (43). These findings were corroborated by yet another study conducted using healthy adults who consumed rice pudding with $1 \mathrm{~g}$, $3 \mathrm{~g}$ or no cinnamon. At the 120-minute mark, AUC was significantly lower following $3 \mathrm{~g}$ cinnamon intake compared to either of the other two conditions; further, postprandial serum insulin was reduced while GLP1 concentrations were increased in the $3 \mathrm{~g}$ cinnamon group (44). Together, these findings suggest that cinnamon is capable of altering glucose homeostasis in healthy adults.

One of the earliest clinical trials in patients with diabetes recruited 60 participants and randomized them into six groups, three of which were treated with 1,3 or $6 \mathrm{~g} /$ day cinnamon while the other three were treated with matching placebo capsules. At the 40-day follow-up point, all doses of cinnamon had produced significantly lower fasting blood glucose levels than the corresponding placebo group. Serum lipids were also more normalized in each of the cinnamon groups 
compared to corresponding controls. Unfortunately, anthropometric measures were not recorded (45). These findings were partially confirmed in a randomized clinical trial in which 79 adults with non-insulin treated type 2 diabetes were randomized to treatment with $3 \mathrm{~g} /$ day cinnamon or a matching placebo. After four months of follow-up, the cinnamon-treated group had a significantly greater reduction-from-baseline in fasting glucose compared to the placebo group; however, neither $\mathrm{HbA} 1 \mathrm{c}$ nor lipid profile differed by treatment assignment (46). By contrast, a clinical trial conducted in 25 postmenopausal women with type 2 diabetes did not detect any by-group difference in fasting glucose, serum lipids or insulin sensitivity. This trial used a $1.5 \mathrm{~g} /$ day cinnamon dose or a placebo for a period of 6 weeks (47).

A UK clinical trial of adults with type 2 diabetes randomized participants to treatment with $2 \mathrm{~g}$ cinnamon or placebo for a duration of 12 weeks. At the end of the follow-up period, participants in the cinnamon group had significant reductions in $\mathrm{HbA} 1 \mathrm{c}$, fasting plasma glucose, waist circumference and BMI. Both systolic and diastolic blood pressure were also significantly reduced compared to the placebo group (48). Systolic blood pressure appeared to be reduced in patients with type 2 diabetes treated with $1.2 \mathrm{~g} /$ day cinnamon vs. placebo; however, when further statistical adjustment was performed, this difference was attributable to regression to the mean in the cinnamon group, and not an actual treatment effect(49).

A study of 204 type 2 diabetes patients randomized participants to one of four conditions in which black tea was supplemented with $3 \mathrm{~g}$ of either cinnamon, saffron, or ginger or an unsupplemented tea serving as a placebo control. After eight weeks, participants in all spicesupplemented groups had significant normalization of lipid profile compared to the placebo group; however, only cinnamon-supplemented participants had significant reductions in fasting blood glucose (50).

Another study randomized participants to take $1 \mathrm{~g} /$ day cinnamon, $1 \mathrm{~g} /$ day whortleberry or placebo supplements for 90 days. Both supplement groups exhibited reductions in measures of glucose homeostasis; however, only the subjects in the cinnamon group had a reduction in BMI (51). Similarly, a randomized clinical trial of consuming $3 \mathrm{~g} /$ day cinnamon vs. a placebo in Asian Indians with metabolic syndrome demonstrated significant reductions in waist circumference, waist-to-hip ratio and $\mathrm{BMI}$ in addition to improved glucose homeostasis in cinnamon-treated vs. placebo treated participants (52).

While individual studies are convincing, metaanalysis forms the ultimate measure of efficacy of an intervention. To that end, a recent meta-analysis evaluated the efficacy of cinnamon on anthropometric and glycemic outcomes. A total of 18 studies were included in the meta-analysis, which confirmed that cinnamon supplementation was associated with significant reductions in fasting blood glucose and HbA1c. Measures of insulin resistance and anthropometric measures including body weight, BMI and waist circumference did not significantly differ between the cinnamon-treated and placebo-treated groups (53).

Despite this analysis, studies of the effect of cinnamon supplementation on anthropometric, glucose homeostasis, lipid profile and hemodynamic measures are planned (54). Improving the quality of intervention studies and the number of participants will eventually provide a definitive answer regarding the clinical efficacy of cinnamon on these parameters.

\section{Capsaicin}

The chemical compound capsaicin (8-methyl- $N$ vanillyl-6-nonenamide) is the active component of chili peppers. It is an alkaloid, hydrophobic, 
colorless, odorless compound, produced as a secondary metabolite, likely as a deterrent against certain herbivores and fungi. Capsaicin is an irritant for mammals, causing a sensation of burning in any tissue with which it comes in contact $(55,56)$.

Cell Studies: Capsaicin is well-recognized as an antiinflammatory, antioxidant, antimicrobial and anticancer compound (57). Additionally, pre-clinical evidence suggests that capsaicin may have antiobesity and anti-diabetes properties (58-64).

In tissue culture, for example, capsaicin efficiently induces apoptosis and inhibits adipogenesis in 3T3-1 preadipocytes and adipocytes $(65,66)$

Animal Studies: Studies in rats have shown that capsaicin decreases body weight. In rats fed a high fat diet supplemented with capsaicin, perirenal adipose tissue weight declined, oxygen consumption was greater and the respiratory quotient (RQ) increased while liver glycogen decreased, leading to metabolic alterations similar to those induced by epinephrine (67).

Administration of a capsaicin analogue to 15week old obese Zucker Diabetic Fatty rats prevented further deterioration of glucose homeostasis. When administered to 19-week-old overtly diabetic Zucker Diabetic Fatty rats, the capsaicin analogue improved glucose tolerance, increased insulin response and reduced dipeptidyl peptidase IV levels in plasma (68). Three-monthold male Wistar rats were fed a high fat obesogenic western diet and gavage-administered hesperidin, capsaicin, both or neither. Capsaicin supplementation decreased body fat gain, prevented insulin resistance, improved blood lipid profile, and prevented non-alcoholic steatohepatitis (69).

Dietary capsaicin has been shown to reduce metabolic dysregulation in obese/diabetic mice by enhancing adiponectin and adiponectin receptor expression. Capsaicin activates transient receptor potential vanilloid-1, which has been shown to prevent adipogenesis through a neurogenic mechanism that modulates fat metabolism via sensory nerves. Mice fed a high fat diet and supplemented with capsaicin had diminished body weight gain, reduced hepatic lipid accumulation and less insulin resistance (66). In male C57BL/6 obese mice fed a high fat diet, capsaicin supplementation lowered fasting glucose, insulin and leptin and prevented impaired glucose tolerance. Additionally, tumor necrosis factoralpha and interleukin (IL)- 6 expression in adipose and liver declined, suggesting that capsaicin mitigates obesity-induced dysregulation of glucose metabolism by enhancing peripheral fatty acid oxidation and reducing systemic inflammation (70).

Human Studies: Epidemiological research indicates that populations that consume capsaicin through food sources have lower obesity prevalence (71). It has been suggested that daily capsaicinoid consumption may facilitate weight management through reductions in energy intake $(72,73)$

Several possible pathways have been identified through which capsaicin may influence body weight in humans. These include energy intake, satiety, energy expenditure, inhibition of adipogenesis and alterations in brown adipose tissue.

Capsaicinoids have been shown to increase glucagon-like peptide 1 (GLP-1), which is associated with weight loss in diabetic individuals, while decreasing ghrelin levels, thus reducing appetite $(74,75)$. Additionally, by stimulating the sympathetic nervous system, specifically via catecholamines, capsaicin has been shown to reduce energy intake and increase energy expenditure (76). 
Mean daily energy intake was significantly lower in both men and women who were randomized to receive either capsaicin capsules or capsaicin delivered in a beverage compared to those who received a placebo. Energy was reduced primarily through reduced fat intake. Satiety also increased in capsaicin-treated participants (72). Consistent with these findings, a systematic review and meta-analysis concluded that ingesting capsaicinoids with meals could reduce energy intake by 74 kcals on average (73).

The effect of capsaicin on energy expenditure was demonstrated in a clinical trial where young adults with obesity were randomized to $2 \mathrm{mg} / \mathrm{day}$ capsaicin or placebo. Subjects in the capsaicin and placebo groups did not differ in terms of energy intake or satiety, nor did they differ in terms of ghrelin, GLP1, peptide YY several hours after consuming their midday meal. However, postprandial resting energy expenditure (REE) increased significantly in the capsaicin group but not in the placebo group (77).

A meta-analysis of studies designed to investigate the efficacy of capsaicin on energy expenditure and respiratory quotient (RQ) identified nine clinical trials of adequate quality. An increase in energy expenditure was observed among participants with $\mathrm{BMI}>25 \mathrm{~kg} / \mathrm{m} 2$, but not in individuals with lower BMI values. The increase in energy expenditure was accompanied by a decrease in $R Q$, indicating enhanced fat oxidation (78). Increased lipid oxidation was also demonstrated in a randomized clinical trial with 91 overweight subjects who had lost weight on a very low-calorie diet that were in a weight maintenance phase. Subjects were randomized to receive $135 \mathrm{mg}$ capsaicin/day or a placebo. The mean \% weight regained during the three-month maintenance period did not significantly differ between treatment groups; however, the increase in RQ was significantly lower in the capsaicin group, demonstrating greater fat oxidation (79).

Yet another pathway through which capsaicin may contribute to weight loss is through alterations to brown adipose tissue (BAT) activity and/or density. In humans, a single oral dose of capsinoids has been shown to increase whole-body energy expenditure, suggesting that capsinoids not only activate but also recruit BAT in humans, meaning that beige cells in white adipose tissue are induced to differentiate into BAT (80-82). This is consistent with animal studies that have shown a capsainoidinduced increase in BAT thermogenesis and body fat mobilization via the sympathetic nervous system $(83,84)$. It has been proposed that capsaicin may influence energy expenditure via non-shivering thermogenesis, though the mechanism is not clear (69).

In summary, studies suggest that adding capsaicinoids to the diet could facilitate weight management, supporting a role for capsaicin as an anti-obesity agent.

\section{Black Pepper}

Piper nigrum (Black pepper) belongs to the Piperaceae family which includes more than 1,000 species with tropical and sub-tropical distribution (85). It is prepared by briefly cooking and drying the unripe fruits (86). Piper nigrum, with its pungent alkaloid piperine as a prominent active ingredient, holds rich phytochemicals that include volatile essential oils, oleoresins, phenolic acids, alkaloids such as chavicine, piperidine and piperetine and other constituents (85). Piper nigrum is widely used in foods for flavor, texture and preservation as well as in traditional medicine in many cultures around the world $(87,88)$. Piper nigrum has multi-targeting potential due to the anti-microbial, antioxidant, anti-inflammatory, anti-proliferative and neuropharmacological activities of its bioactive derivates (86-88). However, the anti-obesity and anti- 
hyperglycemic activities remain largely unexplored in clinical trials $(89,90)$, precluding recommendation of its use for treating obesity. Evidence-based treatment guidelines require more studies in human subjects (85).

Cell studies: Several in-vitro studies have demonstrated that Piper nigrum extracts can modulate thermogenesis, energy homeostasis, lipid accumulation, insulin sensitivity and glucose uptake through various signaling pathways (90-98). Because of these effects, Piper nigrum has been identified as a potential intervention for the treatment of obesity and type 2 diabetes (90-98).

Piper nigrum extract and piperine have been shown to inhibit adipogenesis by down-regulating many genes associated with lipid accumulation during the differentiation of 3T3-L1 pre-adipocyte cells. Simultaneously, Piper nigrum has been shown to up-regulate various genes associated with lipid catabolic process (92).

Trans-pellitorine, an alkamide present in Piper nigrum, has been shown to reduce lipid accumulation at the intermediate stages of adipogenesis and by inhibiting short-term fatty acid uptake in 3T3-L1 pre-adipocytes, likely through several signaling pathways (93). Piper nigrum extract increased glucose uptake by more than $200 \%$ after the induction of insulin and suppressed lipid accumulation in a concentration-dependent manner in 3T3-L1 adipocytes cells (94).

Alkamides isolated from Piper nigrum showed a significant inhibitory effect on the activity of rat liver microsomal acyl CoA diacylglycerol acyltransferase enzyme, a microsomal enzyme that plays a fundamental role in triglyceride synthesis (91). Piperine has been shown to increase ATPase activity in an in-vitro rabbit muscle assay by destabilizing the super-relaxed state of myosin heads, an effect which may enhance thermogenesis of resting muscles (90). When Indonesian medicinal plants were screened for their ability to inhibit the activity of protein tyrosine phosphatase 1B (PTP1B), a negative regulator of the leptin and insulin signaling pathways, water extract of Piper nigrum exhibited more than $70 \%$ inhibition at a concentration of $25 \mu \mathrm{g} / \mathrm{mL}$ (95). The ion channels transient receptor potential vanilloid 1 (TRPV1) and transient receptor potential ankyrin 1 (TRPA1) are involved in a wide range of processes, including energy and glucose homeostasis $(31,99)$ and possibly appetite (100). Several piperine analogs from Piper nigrum were found to be TRPV1 and TRPA1 agonists in human emryonic kidney 293 cells which expressed TRPV1 or TRPA1 (96-98).

Animal studies: Rodents fed a high-fat diet (HFD) developed abdominal obesity (101). Previous studies have shown that increased adiposity leads to a systemic pro-inflammatory response $(102,103)$ that seems to promote and accentuate metabolic abnormalities $(103,104)$.

Piper nigrum extracts have been shown to have anti-obesity and anti-hyperglycemic activities in both HFD-induced obese rats' models $(89,101$, 105-110) and high carbohydrate, high fat diet (HCHF)-induced obese rats' models $(111,112)$. Oral administration of $200 \mathrm{mg} / \mathrm{kg}$ ethyl acetate or aqueous extracts of Piper nigrum for 42 days markedly reduced the body weight, $\%$ fat, fat-free mass, glucose profile, lipid profile, liver marker enzymes and leptin, inhibited the activity of pancreatic lipase and increased adiponectin of HFD fed Sprague Dawley rats (101). Dietary intake of piperine $(0.02 \%)$ for 8 weeks decreased body weight as well as weight of adipose tissue in HFD fed Wistar rats (110). Moreover, piperine intake has been shown to enhance digestive enzyme activity and promoted bile secretion; however, key hepatic lipogenic enzyme activity was concomitantly reduced (110). Thus, although consumption of piperine along with a HFD may facilitate the 
digestion and absorption of dietary fats, it may also down-regulate lipogenesis in the body (110).

Supplementing with HFD-fed Sprague Dawley rats with piperine $(40 \mathrm{mg} / \mathrm{kg})$ for 3 weeks resulted in reduced body weight, fat mass and improved lipid profile, with no change in food intake (109). In HFD-fed C57BL/6N mice supplemented with piperine $(0.05 \%)$ for 10 weeks, body weight, visceral fat-pad, liver weight, plasma and hepatic lipid levels were all decreased while insulin sensitivity was increased (108). Moreover, dietary piperine decreased the mRNA expression of $L X R \alpha$, a nuclear receptor that regulates the metabolism of several important lipids and also decreased the mRNA expression of key gluconeogenic enzymes, lipogenic transcription factors and their target genes in HFDfed mice (108). Supplementation of piperonal (20, 30 and $40 \mathrm{mg} / \mathrm{kg}$ ) for 42 days attenuated HFDinduced body weight, body fat and biochemical changes through normalization of amylase and lipase levels, modulation of key lipid metabolizing and obesogenic genes in Sprague Dawley rats (89).

Similarly, piperine supplementation in different doses $(20,30$ and $40 \mathrm{mg} / \mathrm{kg}$ ) for 42 days suppressed body weight and leptin, normalized the elevated levels of amylase and lipase and improved adiposity index, insulin sensitivity and adiponectin levels in HFD-fed Sprague Dawley rats (107). Administration of piperine $(50 \mathrm{mg} / \mathrm{kg})$ for 28 days to C57BL/6N mice appeared to reverse preexisting HFD-induced weight gain, hepatic steatosis and insulin resistance, likely mediated by adiponectinAMPK signaling pathway activation (106). Piperine supplementation ( $30 \mathrm{mg} / \mathrm{kg}$ ) for 16 weeks reduced body weight, abdominal circumference and abdominal fat pads and improved the impaired oral glucose tolerance, blood pressure, liver function, oxidative stress and markers of inflammation in HCHF-fed Wistar rats (111). The addition of $0.03 \%$ and $0.05 \%$ of piperine or $1.0 \%$ of Piper nigrum decreased the visceral body fat accumulation in
C57BL/6J mice that were fed a high fat and high sucrose diet (112). These effects were at least partially mediated through increased expression of thermogenic protein uncoupling protein-1 (112). The suppressive effects of Piper nigrum was higher than that of piperine alone, implying that additional components are involved in fat accumulation (112). HFD-fed C57BL/6 mice exposed to caloric restriction supplemented with curcumin + piperine lost more fat and had significantly lower inflammatory markers (i.e. IL-1 $\beta$ and $K C / G R O$ ) concentrations in plasma, compared to control groups (105). These findings are not uniform, however. In another study of HFD-fed C57BL/6 mice, supplementation of a calorie restricted diet with curcumin and/or piperine $(50 \mathrm{mg} / \mathrm{kg})$ for 5 weeks provided no additional benefit to weight loss; on the contrary, the effect of calorie restriction $\mathrm{T}$ cell-mediated function was blunted by supplementation (113).

Human studies: Few human studies have investigated the effect of Piper nigrum on weight management, energy balance and appetite regulation (114-120). Moreover, in most of the relevant literature Piper nigrum extracts were combined with other food ingredients (115-118) that were most likely to enhance their bioavailability (85).

In a double-blind, randomized, placebocontrolled trial (RCT), investigators examined a combination of bioactive food ingredients (capsaicinoids, epigallocatechin gallate, piperin from Piper nigrum, and L-carnitine) on changes in appetite control, energy expenditure and serum markers of lipolysis in 37 overweight subjects. The supplemented group experienced a significantly greater increase in satiety (116). The same research group randomized 86 overweight subjects to this same combination of bioactive food ingredients or placebo. At the 8-week follow-up point, 
supplemented subjects had decreased insulin resistance and inflammatory adipokines and increased resting energy expenditure (115).

Another RCT was conducted in 40 obese women who were randomized to three months supplementation with $150 \mathrm{mg} /$ dose of Greenselect Phytosome ${ }^{\circledR}$ combined with a $15 \mathrm{mg} /$ dose of piperine from Piper nigrum or placebo for weight maintenance after weight loss achieved by 3-month lifestyle intervention. Compared to the placebo group, women treated with the supplement had less weight regain and thus maintained their reduced body weight (117).

In a pair matched, double-blind RCT, 30 participants were randomized to a multi-ingredient performance supplement including peperine or placebo during 6 weeks of resistance training. Both groups exhibited improvements in body composition with no by-treatment difference in outcome (118).

A cross-over RCT in 16 healthy subjects was designed to evaluate whether the consumption of a Piper nigrum-based beverage would effect postprandial glycaemia, appetite, gut hormones, thyroid function, and gastrointestinal well-being after a white wheat bread challenge meal (50 gr available carbohydrates). The Piper nigrum-based beverage modulated overall appetite by increasing satiety, no other outcomes differed by treatment group; however, the study beverage did not contain piperine due to its low water solubility and melting point (119).

In a single-blind, cross-over, RCT, 22 young normal-weight males were assigned to receive a brunch meal with either pepper (1.3 g), ginger (20 g), horseradish ( $8.3 \mathrm{~g})$, mustard ( $21 \mathrm{~g}$ ) or no spices (placebo). Each test day was separated by a >3week washout period. No reliable treatment effects of Piper nigrum on appetite, energy intake or energy balance were observed (114). This aligns with the findings of another cross-over RCT in which overweight postmenopausal women were randomized to the addition of $0.5 \mathrm{mg}$ Piper nigrum per meal or not. Under eucaloric conditions, the addition of the Piper nigrum was not associated with changes in glucose or insulin response or gut hormone levels; further, Piper nigrum did not modify 24-hour energy expenditure measured by indirect calorimeter, (120).

\section{Curcumin}

Curcumin is the principal lipophilic polyphenol present in the rhizome of turmeric plant Curcuma longa $L$. (common names Turmeric, Indian Saffron). Curcumin belongs to the ginger family and has long been used as culinary spice (121). Traditionally, it has been used in Ayurveda medicines and other traditional medicine systems across Asia to treat various diseases such as asthma, anorexia, coughing, hepatic diseases, diabetes, heart disease, and Alzheimer's disease (122). Chemical analysis of turmeric has yielded essential oil, fatty oil, and moisture. The pale yellow to orange oil is composed of a number of monoterpenes and sesquiterpenes, including zingiberene, curcumene, $\alpha$ and $\beta$ turmerone, and curlone amongst others. The yellow pigments are curcuminoids consisting of curcumin (di-feruloyl-methane), phydroxycinnamoyl (feruloyl) methane and $p, p^{\prime}-$ dihydroxy-dicinnamoyl-methane in three to five per cent concentration (123). Despite promising in vitro results, poor aqueous solubility, diverse bioavailability, and pharmacokinetic profiles limit curcumin's therapeutic usage. To address these issues, several curcumin formulations have been developed (124). However, suboptimal sample preparation and analysis methodologies often hamper the accurate evaluation of bioactivities and their clinical efficacy (125) and analytical methods for determination of curcuminoids in turmeric are still in debate (126). 
Cell studies: Recent cellular studies indicate that curcumin can influence signal transduction and gene expression, thus reducing the viability of adipocytes and the proliferation of preadipocytes, the suppression of adipocyte differentiation and triglyceride accumulation, the stimulation of lipolysis and fatty acid $\beta$-oxidation and reduced inflammation (127). Alongside this, the polyphenols modulate signaling pathways including the adenosine-monophosphate-activated protein kinase, peroxisome proliferator activated receptor $\gamma$, CCAAT/enhancer binding protein $\alpha$, peroxisome proliferator activator receptor gamma activator 1-alpha, sirtuin 1, sterol regulatory element binding protein-1c, uncoupling proteins 1 and 2, and nuclear factor-kB that regulate adipogenesis, antioxidant and anti-inflammatory responses (127). Curcumin has also been found to effect sterol regulatory element-binding proteins (SREBPs), major transcription factors regulating the expression of genes involved in the biosynthesis of cholesterol, fatty acid and triglyceride.

The antioxidant and anti-inflammatory properties of curcumin are related to improved arterial health. Mechanistic studies have revealed superoxide dismutase, heme oxygenase-1 and nuclear factor erythroid 2-related factor 2 as emerging targets for the beneficial effects of curcumin on the vasculature (128).

Animal studies: Animal studies strongly suggest that polyphenols from curcumin have a pronounced effect on obesity as evidenced by lower body weight, fat mass and triglycerides. This appears to be mediated through enhanced energy expenditure and fat utilization, together with modulated glucose hemostasis (127). High fat diet fed male C57BL/ 6 were administered curcumin (80 and $40 \mathrm{mg} / \mathrm{kg} /$ day) for 12 weeks. Curcumin treatment significantly reduced body weight gain by $20.7 \%$ at the $80 \mathrm{mg} / \mathrm{kg}$ dose (129). In addition, at a concentration of $10 \mu \mathrm{M}$, curcumin remarkably down-regulated mRNA expressions of SREBP target genes including nine fatty acid and triglyceride synthesis-related genes (SREBP-1, fatty acid synthase (FAS), acetyl-Coenzyme A carboxylase alpha (ACC1), stearoyl-Coenzyme $A$ desaturase 1 (SCD-1), SCD-2, ATP citrate lyase (ACL), fatty acid desaturase 1 (FADS1), FADS2 and glycerol-3phosphate acyltransferase (GPAT)) and eleven cholesterol synthesis related genes (SREBP-2, 3hydroxy-3-methylglutaryl-Coenzyme A reductase (HMGCR), 3-hydroxy-3-methylglutaryl-Coenzyme A synthase (HMGCS), lanosterol synthase (LSS), sterol-C4-methyl oxidase-like (Sc4mol), squalene epoxidase (SE), 24-dehydrocholesterol reductase (DHCR24), 7-dehydrocholesterol reductase (DHCR7), farnesyl (129).

Additional studies in the same male mice models have shown that HFD-feeding supplemented with curcumin $(4 \mathrm{~g} / \mathrm{kg}$ diet added 2 days/week) prevented body weight gain compared to non-supplemented HFD-fed controls or low-fat fed mice (LFD). HFD feeding increased the fasting plasma insulin levels but dietary curcumin supplementation significantly prevented this increase. Although HFD reduced and curcumin increased plasma adiponectin levels, the differences did not reach statistical significance (130). In addition, curcumin supplemented HFD has been shown to improve insulin signaling and inhibited lipogenic gene expression in the liver (130, 131). Moreover, curcumin has been shown to promote browning of white adipose tissue in dietinduced obese mice, which suggests increased energy expenditure as another mechanism through which curcumin supplementation reduces fat mass deposition (132).

Human studies: Among obese individuals, RCTs have shown that a supplementation of $600-1000 \mathrm{mg}$ curcumin a day for 4-8 weeks generated a reduction 
in pro-inflammatory cytokines such as TNF- $\alpha$, IL-6, TGF- $\beta$ and MCP-1 among obese individuals in comparison to placebo $(133,134)$. A decrease in IL6 , hs-CRP and oxidative stress levels has also been shown among adolescent overweight girls who consumed $500 \mathrm{mg}$ curcumin during 10 weeks of a weight loss diet (135). A recent comprehensive review concluded that curcumin can effectively inhibit multiple signaling molecules involved in inflammation including NF-KB, JAKs/STATs, MAPKs, $\beta$-catenin, and Notch-1(136). Despite the metabolic advantages, no weight reduction has been documented in these studies.

\section{CONCLUSIONS}

Herbs, spices and their derivative nutrition supplements are commonly incorporated into the treatment arsenal for weight management. Preclinical studies demonstrate mechanisms through which these herbs exert their beneficial metabolic effects, providing proof of concept. However, welldesigned, adequately powered clinical trials are necessary before treatment guidelines for these products can be developed.

\section{REFERENCES}

1. Milano W, De Biasio V, Di Munzio W, et al. Obesity: the New Global Epidemic. Pharmacological Treatment, Opportunities and Limits for Personalized Therapy. Endocr Metab Immune Disord Drug Targets 2020; 15(10): 1871530320666200515112853.

2. Talukdar D, Seenivasan S, Cameron AJ, et al. The association between national income and adult obesity prevalence: Empirical insights into temporal patterns and moderators of the association using 40 years of data across 147 countries. PLOS One 2020;15(5).

3. Stoner L, Cornwall J. Did the American Medical Association make the correct decision classifying obesity as a disease? Australas Med J 2014;7(11):4624.

4. Leitner DR, Frühbeck G, Yumuk V, et al. Obesity and Type 2 Diabetes: Two Diseases with a Need for Combined Treatment Strategies - EASO Can Lead the Way. Obes Facts 2017;10(5):483-92.

5. Seravalle G, Grassi G. Obesity and hypertension. Pharmacol Res 2017;122:1-7.

6. Censin JC, Peters SAE, Bovijn J, et al. Causal relationships between obesity and the leading causes of death in women and men. PLoS Genet 2019;15(10).

7. Avgerinos KI, Spyrou N, Mantzoros CS, et al. Obesity and cancer risk: Emerging biological mechanisms and perspectives. Metabolism 2019;92:121-35.

8. Yumuk V, Tsigos C, Fried M, et al. European Guidelines for Obesity Management in Adults. Obes Facts $2015 ; 8(6): 402-24$.

9. Eldalo AS, Alotaibi MN, Alenazi TO, et al. Use of Herbal Medicines in the Treatment of Obesity in Taif, Saudi Arabia. Saudi J Med Med Sci 2017;5(2):149-54.

10. Farrington R, Musgrave IF, Byard RW. Evidence for the efficacy and safety of herbal weight loss preparations. J Integr Med 2019;17(2):87-92.

11. Brown AC. An overview of herb and dietary supplement efficacy, safety and government regulations in the United States with suggested improvements. Part 1 of 5 series. Food Chem Toxicol 2017;107(Pt A):449-71

12. Kumar S, Kumari R, Mishra S. Pharmacological properties and their medicinal uses of Cinnamomum: a review. J Pharm Pharmacol 2019;71(12):1735-61.

13. Ahmad S, Hassan A, Abbasi WM, et al. Phytochemistry and pharmacological potential of Cassia absus - a review. J Pharm Pharmacol 2018;70(1):27-41.

14. Suzuki H, Inadomi JM, Hibi T. Japanese herbal medicine in functional gastrointestinal disorders. Neurogastroenterol Motil 2009;21(7):688-96.

15. Flores $\mathrm{KE}$, Quinlan $\mathrm{MB}$. Ethnomedicine of menstruation in rural Dominica, West Indies. J Ethnopharmacol 2014;153(3):624-34. 
16. Rambod M, Nazarinia M, Raieskarimian F. The prevalence and predictors of herbal medicines usage among adult rheumatoid arthritis patients: A casecontrol study. Complement Ther Med 2018;41:220-4.

17. Ferro TAF, Souza EB, Suarez MAM, et al. Topical Application of Cinnamaldehyde Promotes Faster Healing of Skin Wounds Infected with Pseudomonas aeruginosa. Molecules 2019;24(8).

18. Banjari I, Misir A, Pavlić M, et al. Traditional Herbal Medicines for Diabetes Used in Europe and Asia: Remedies From Croatia and Sri Lanka. Altern Ther Health Med 2019;25(3):40-52.

19. Mielecki M, Lesyng B. Cinnamic Acid Derivatives as Inhibitors of Oncogenic Protein Kinases--Structure, Mechanisms and Biomedical Effects. Curr Med Chem 2016;23(10):954-82.

20. Dhara L, Tripathi A. Cinnamaldehyde: a compound with antimicrobial and synergistic activity against ESBL-producing quinolone-resistant pathogenic Enterobacteriaceae. Eur J Clin Microbiol Infect Dis 2020;39(1):65-73

21. Chen BJ, Fu CS, Li GH, et al. Cinnamaldehyde Analogues as Potential Therapeutic Agents. Mini Rev Med Chem 2017;17(1):33-43.

22. Fujisawa S, Murakami Y. Eugenol and Its Role in Chronic Diseases. Adv Exp Med Biol 2016;929:45-66

23. Riveiro ME, De Kimpe N, Moglioni A, et al. Coumarins: old compounds with novel promising therapeutic perspectives. Curr Med Chem 2010;17(13):1325-38.

24. Hariri M, Ghiasvand R. Cinnamon and Chronic Diseases. Adv Exp Med Biol 2016;929:1-24.

25. Kyle TK, Dhurandhar EJ, Allison DB. Regarding Obesity as a Disease: Evolving Policies and Their Implications. Endocrinol Metab Clin North Am 2016;45(3):511-20.

26. Khan A, Bryden NA, Polansky MM, et al. Insulin potentiating factor and chromium content of selected foods and spices. Biol Trace Elem Res $1990 ; 24(3): 183-8$.

27. Imparl-Radosevich J, Deas S, Polansky MM, et al. Regulation of PTP-1 and insulin receptor kinase by fractions from cinnamon: implications for cinnamon regulation of insulin signalling. Horm Res 1998;50(3):177-82.

28. Jarvill-Taylor KJ, Anderson RA, Graves DJ. A hydroxychalcone derived from cinnamon functions as a mimetic for insulin in 3T3-L1 adipocytes. J Am Coll Nutr 2001;20(4):327-36.

29. Medagama AB. The glycaemic outcomes of Cinnamon, a review of the experimental evidence and clinical trials. Nutr J 2015;14(108):015-0098.

30. Højlund K. Metabolism and insulin signaling in common metabolic disorders and inherited insulin resistance. Dan Med J 2014;61(7).

31. Derbenev AV, Zsombok A. Potential therapeutic value of TRPV1 and TRPA1 in diabetes mellitus and obesity. Seminars in immunopathology 2016;38(3):397-406.

32. Hafizur RM, Hameed A, Shukrana M, et al. Cinnamic acid exerts anti-diabetic activity by improving glucose tolerance in vivo and by stimulating insulin secretion in vitro. Phytomedicine 2015;22(2):297-300

33. Uchiyama S, Taniguchi Y, Saka A, et al. Prevention of diet-induced obesity by dietary black tea polyphenols extract in vitro and in vivo. Nutrition 2011;27(3):28792.

34. Ogasawara J, Kitadate K, Nishioka H, et al. Oligonol, an oligomerized lychee fruit-derived polyphenol, activates the Ras/Raf-1/MEK1/2 cascade independent of the IL- 6 signaling pathway in rat primary adipocytes. Biochem Biophys Res Commun 2010;402(3):554-9

35. Jiang J, Emont MP, Jun $\mathrm{H}$, et al. Cinnamaldehyde induces fat cell-autonomous thermogenesis and metabolic reprogramming. Metabolism 2017;77:5864.

36. Lu Z, Jia $Q$, Wang $R$, et al. Hypoglycemic activities of A- and B-type procyanidin oligomer-rich extracts from different Cinnamon barks. Phytomedicine 2011;18(4):298-302.

37. Sartorius $\mathrm{T}$, Peter A, Schulz N, et al. Cinnamon extract improves insulin sensitivity in the brain and lowers liver fat in mouse models of obesity. PLoS One 2014;9(3). 
38. Couturier K, Qin B, Batandier C, et al. Cinnamon increases liver glycogen in an animal model of insulin resistance. Metabolism 2011;60(11):1590-7.

39. Qin B, Nagasaki $M$, Ren $M$, et al. Cinnamon extract prevents the insulin resistance induced by a highfructose diet. Horm Metab Res 2004;36(2):119-25.

40. Shalaby MA, Saifan HY. Some pharmacological effects of cinnamon and ginger herbs in obese diabetic rats. J Intercult Ethnopharmacol 2014;3(4):144-9.

41. Van Hul M, Geurts L, Plovier H, et al. Reduced obesity, diabetes, and steatosis upon cinnamon and grape pomace are associated with changes in gut microbiota and markers of gut barrier. Am J Physiol Endocrinol Metab 2018;314(4):E334-E52.

42. Solomon TP, Blannin AK. Effects of short-term cinnamon ingestion on in vivo glucose tolerance. Diabetes Obes Metab 2007;9(6):895-901.

43. Solomon TP, Blannin AK. Changes in glucose tolerance and insulin sensitivity following 2 weeks of daily cinnamon ingestion in healthy humans. Eur $J$ Appl Physiol 2009;105(6):969-76.

44. Hlebowicz J, Hlebowicz A, Lindstedt S, et al. Effects of 1 and $3 \mathrm{~g}$ cinnamon on gastric emptying, satiety, and postprandial blood glucose, insulin, glucosedependent insulinotropic polypeptide, glucagon-like peptide 1, and ghrelin concentrations in healthy subjects. Am J Clin Nutr 2009;89(3):815-21.

45. Khan A, Safdar M, Ali Khan MM, et al. Cinnamon improves glucose and lipids of people with type 2 diabetes. Diabetes Care 2003;26(12):3215-8.

46. Mang B, Wolters M, Schmitt B, et al. Effects of a cinnamon extract on plasma glucose, $\mathrm{HbA}$, and serum lipids in diabetes mellitus type 2. Eur J Clin Invest $2006 ; 36(5): 340-4$

47. Vanschoonbeek K, Thomassen BJ, Senden JM, et al. Cinnamon supplementation does not improve glycemic control in postmenopausal type 2 diabetes patients. J Nutr 2006;136(4):977-80.

48. Akilen R, Tsiami A, Devendra D, et al. Glycated haemoglobin and blood pressure-lowering effect of cinnamon in multi-ethnic Type 2 diabetic patients in the UK: a randomized, placebo-controlled, doubleblind clinical trial. Diabet Med 2010;27(10):1159-67.

49. Wainstein J, Stern N, Heller S, et al. Dietary cinnamon supplementation and changes in systolic blood pressure in subjects with type 2 diabetes. J Med Food 2011;14(12):1505-10

50. Azimi P, Ghiasvand R, Feizi A, et al. Effects of Cinnamon, Cardamom, Saffron, and Ginger Consumption on Markers of Glycemic Control, Lipid Profile, Oxidative Stress, and Inflammation in Type 2 Diabetes Patients. Rev Diabet Stud 2014;11(3-4):25866.

51. Mirfeizi M, Mehdizadeh Tourzani Z, Mirfeizi SZ, et al. Controlling type 2 diabetes mellitus with herbal medicines: A triple-blind randomized clinical trial of efficacy and safety. J Diabetes 2016;8(5):647-56.

52. Gupta Jain S, Puri S, Misra A, et al. Effect of oral cinnamon intervention on metabolic profile and body composition of Asian Indians with metabolic syndrome: a randomized double -blind control trial. Lipids Health Dis 2017;16(1):017-0504.

53. Namazi N, Khodamoradi K, Khamechi SP, et al. The impact of cinnamon on anthropometric indices and glycemic status in patients with type 2 diabetes: A systematic review and meta-analysis of clinical trials. Complement Ther Med 2019;43:92-101.

54. Neto J, Damasceno MMC, Ciol MA, et al. Analysis of the effectiveness of cinnamon (Cinnamomum verum) in the reduction of glycemic and lipidic levels of adults with type 2 diabetes: A study protocol. Medicine 2020;99(1):0000000000018553.

55. Thiele R, Mueller-Seitz E, Petz M. Chili pepper fruits: presumed precursors of fatty acids characteristic for capsaicinoids. J Agric Food Chem 2008;56(11):421924.

56. Whiting S, Derbyshire E, Tiwari BK. Capsaicinoids and capsinoids. A potential role for weight management? A systematic review of the evidence. Appetite 2012;59(2):341-8. 
57. Sharma SK, Vij AS, Sharma M. Mechanisms and clinical uses of capsaicin. Eur J Pharmacol 2013;720(1-3):55-62.

58. Alkhatib A, Seijo M, Larumbe E, et al. Acute effectiveness of a "fat-loss" product on substrate utilization, perception of hunger, mood state and rate of perceived exertion at rest and during exercise. J Int Soc Sports Nutr 2015;12(44):015-0105.

59. Baskaran P, Krishnan V, Fettel K, et al. TRPV1 activation counters diet-induced obesity through sirtuin-1 activation and PRDM-16 deacetylation in brown adipose tissue. Int J Obes 2017;41(5):739-49.

60. Josse AR, Sherriffs SS, Holwerda AM, et al. Effects of capsinoid ingestion on energy expenditure and lipid oxidation at rest and during exercise. Nutr Metab 2010;7(65):1743-7075.

61. Kobayashi Y, Nakano Y, Kizaki M, et al. Capsaicin-like anti-obese activities of evodiamine from fruits of Evodia rutaecarpa, a vanilloid receptor agonist. Planta Med 2001;67(7):628-33.

62. Westerterp-Plantenga MS, Smeets A, Lejeune MP. Sensory and gastrointestinal satiety effects of capsaicin on food intake. Int J Obes 2005;29(6):682-8.

63. Yoshioka M, St-Pierre S, Drapeau V, et al. Effects of red pepper on appetite and energy intake. Br J Nutr 1999;82(2):115-23.

64. Zheng J, Zheng S, Feng Q, et al. Dietary capsaicin and its anti-obesity potency: from mechanism to clinical implications. Biosci Rep 2017;37(3):30.

65. Hsu CL, Yen GC. Effects of capsaicin on induction of apoptosis and inhibition of adipogenesis in 3T3-L1 cells. J Agric Food Chem 2007;55(5):1730-6.

66. Leung FW. Capsaicin as an anti-obesity drug. Prog Drug Res 2014;68:171-9.

67. Kawada T, Watanabe T, Takaishi T, et al. Capsaicininduced beta-adrenergic action on energy metabolism in rats: influence of capsaicin on oxygen consumption, the respiratory quotient, and substrate utilization. Proc Soc Exp Biol Med 1986;183(2):250-6.

68. Gram DX, Hansen AJ, Deacon CF, et al. Sensory nerve desensitization by resiniferatoxin improves glucose tolerance and increases insulin secretion in Zucker Diabetic Fatty rats and is associated with reduced plasma activity of dipeptidyl peptidase IV. Eur J Pharmacol 2005;509(2-3):211-7.

69. Mosqueda-Solís A, Sánchez J, Reynés B, et al. Author Correction: Hesperidin and capsaicin, but not the combination, prevent hepatic steatosis and other metabolic syndrome-related alterations in western diet-fed rats. Sci Rep. 2020 Feb 18;10(1):3218. doi: $10.1038 / s 41598-020-60110-6$.

70. Kang JH, Goto T, Han IS, et al. Dietary capsaicin reduces obesity-induced insulin resistance and hepatic steatosis in obese mice fed a high-fat diet. Obesity 2010;18(4):780-7.

71. Wahlqvist ML, Wattanapenpaiboon N. Hot foods-unexpected help with energy balance? Lancet 2001;358(9279):348-9.

72. Ludy MJ, Mattes RD. The effects of hedonically acceptable red pepper doses on thermogenesis and appetite. Physiol Behav 2011;102(3-4):251-8.

73. Whiting S, Derbyshire EJ, Tiwari B. Could capsaicinoids help to support weight management? A systematic review and meta-analysis of energy intake data. Appetite 2014;73:183-8.

74. Josse AR, Sherriffs SS, Holwerda AM, et al. Effects of capsinoid ingestion on energy expenditure and lipid oxidation at rest and during exercise. Nutr Metab (Lond) 2010;7:65.

75. Smeets AJ, Westerterp-Plantenga MS. The acute effects of a lunch containing capsaicin on energy and substrate utilisation, hormones, and satiety. Eur $J$ Nutr 2009;48(4):229-34.

76. Hursel R, Westerterp-Plantenga MS. Thermogenic ingredients and body weight regulation. Int J Obes 2010;34(4):659-69.

77. Rigamonti AE, Casnici C, Marelli O, et al. Acute administration of capsaicin increases resting energy expenditure in young obese subjects without affecting energy intake, appetite, and circulating levels of orexigenic/anorexigenic peptides. Nutr Res 2018;52:71-9. 
78. Zsiborás C, Mátics R, Hegyi P, et al. Capsaicin and capsiate could be appropriate agents for treatment of obesity: A meta-analysis of human studies. Crit Rev Food Sci Nutr 2018;58(9):1419-27.

79. Lejeune MP, Kovacs EM, Westerterp-Plantenga MS. Effect of capsaicin on substrate oxidation and weight maintenance after modest body-weight loss in human subjects. Br J Nutr 2003;90(3):651-59.

80. Yoneshiro T, Aita S, Kawai Y, et al. Nonpungent capsaicin analogs (capsinoids) increase energy expenditure through the activation of brown adipose tissue in humans. Am J Clin Nutr 2012;95(4):845-50.

81. Yoneshiro T, Aita S, Matsushita M, et al. Recruited brown adipose tissue as an antiobesity agent in humans. J Clin Invest 2013;123(8):3404-8.

82. Nirengi $\mathrm{S}$, Homma $\mathrm{T}$, Inoue $\mathrm{N}$, et al. Assessment of human brown adipose tissue density during daily ingestion of thermogenic capsinoids using nearinfrared time-resolved spectroscopy. J Biomed Opt 2016;21(9):091305.

83. lida $\mathrm{T}$, Moriyama $\mathrm{T}$, Kobata $\mathrm{K}$, et al. TRPV1 activation and induction of nociceptive response by a nonpungent capsaicin-like compound, capsiate. Neuropharmacology 2003;44(7):958-67.

84. Ono K, Tsukamoto-Yasui M, Hara-Kimura $\mathrm{Y}$, et al. Intragastric administration of capsiate, a transient receptor potential channel agonist, triggers thermogenic sympathetic responses. J Appl Physiol $1985 ; 110(3): 789-98$.

85. Butt MS, Pasha I, Sultan MT, et al. Black pepper and health claims: a comprehensive treatise. Critical reviews in food science and nutrition 2013;53(9):87586.

86. Meghwal M, Goswami TK. Piper nigrum and piperine: an update. Phytotherapy research : PTR 2013;27(8):1121-30.

87. Shityakov $S$, Bigdelian $E$, Hussein $A A$, et al. Phytochemical and pharmacological attributes of piperine: A bioactive ingredient of black pepper. European journal of medicinal chemistry 2019;176:149-61
88. Salehi B, Zakaria ZA, Gyawali R, et al. Piper Species: A Comprehensive Review on Their Phytochemistry, Biological Activities and Applications. Molecules (Basel, Switzerland) 2019;24(7).

89. Meriga B, Parim B, Chunduri VR, et al. Antiobesity potential of Piperonal: promising modulation of body composition, lipid profiles and obesogenic marker expression in HFD-induced obese rats. Nutrition \& metabolism 2017;14:72.

90. Nogara L, Naber N, Pate E, et al. Piperine's mitigation of obesity and diabetes can be explained by its upregulation of the metabolic rate of resting muscle. Proceedings of the National Academy of Sciences of the United States of America 2016;113(46):13009-14.

91. Lee SW, Rho MC, Park HR, et al. Inhibition of diacylglycerol acyltransferase by alkamides isolated from the fruits of Piper longum and Piper nigrum. Journal of agricultural and food chemistry 2006;54(26):9759-63.

92. Park UH, Jeong HS, Jo EY, et al. Piperine, a component of black pepper, inhibits adipogenesis by antagonizing PPARgamma activity in 3T3-L1 cells. Journal of agricultural and food chemistry 2012;60(15):3853-60.

93. Lieder B, Zaunschirm M, Holik AK, et al. The Alkamide trans-Pellitorine Targets PPARgamma via TRPV1 and TRPA1 to Reduce Lipid Accumulation in Developing 3T3-L1 Adipocytes. Frontiers in pharmacology 2017;8:316.

94. Lahrita L, Kato E, Kawabata J. Uncovering potential of Indonesian medicinal plants on glucose uptake enhancement and lipid suppression in 3T3-L1 adipocytes. Journal of ethnopharmacology 2015;168:229-36

95. Saifudin A, Kadota S, Tezuka Y. Protein tyrosine phosphatase 1B inhibitory activity of Indonesian herbal medicines and constituents of Cinnamomum burmannii and Zingiber aromaticum. Journal of natural medicines 2013;67(2):264-70.

96. Watanabe T, Terada Y. Food Compounds Activating Thermosensitive TRP Channels in Asian Herbal and 
Medicinal Foods. Journal of nutritional science and vitaminology 2015;61 Suppl:S86-8.

97. Okumura $Y$, Narukawa $M$, Iwasaki $Y$, et al. Activation of TRPV1 and TRPA1 by black pepper components. Bioscience, biotechnology, and biochemistry $2010 ; 74(5): 1068-72$.

98. McNamara FN, Randall A, Gunthorpe MJ. Effects of piperine, the pungent component of black pepper, at the human vanilloid receptor (TRPV1). British journal of pharmacology 2005;144(6):781-90.

99. Christie S, Wittert GA, Li H, et al. Involvement of TRPV1 Channels in Energy Homeostasis. Frontiers in endocrinology 2018;9:420.

100. Wang P, Yan Z, Zhong J, et al. Transient receptor potential vanilloid 1 activation enhances gut glucagon-like peptide-1 secretion and improves glucose homeostasis. Diabetes 2012;61(8):2155-65.

101. Parim B, Harishankar N, Balaji M, et al. Effects of Piper nigrum extracts: Restorative perspectives of high-fat diet-induced changes on lipid profile, body composition, and hormones in Sprague-Dawley rats. Pharmaceutical biology 2015;53(9):1318-28.

102. Scherer PE. Adipose tissue: from lipid storage compartment to endocrine organ. Diabetes 2006;55(6):1537-45.

103. Christensen MHE, Fadnes DJ, Rost TH, et al. Inflammatory markers, the tryptophan-kynurenine pathway, and vitamin B status after bariatric surgery. 2018;13(2):e0192169.

104. Goktas Z, Moustaid-Moussa N, Shen CL, et al. Effects of bariatric surgery on adipokine-induced inflammation and insulin resistance. Frontiers in endocrinology 2013;4:69.

105. Miyazawa T, Nakagawa K, Kim SH, et al. Curcumin and piperine supplementation of obese mice under caloric restriction modulates body fat and interleukin-1beta. Nutrition \& metabolism 2018;15:12

106. Choi S, Choi Y, Choi Y, et al. Piperine reverses high fat diet-induced hepatic steatosis and insulin resistance in mice. Food chemistry 2013;141(4):3627-35.
107. BrahmaNaidu $P$, Nemani $H$, Meriga $B$, et al. Mitigating efficacy of piperine in the physiological derangements of high fat diet induced obesity in Sprague Dawley rats. Chemico-biological interactions 2014;221:42-51.

108. Jwa H, Choi Y, Park UH, et al. Piperine, an LXRalpha antagonist, protects against hepatic steatosis and improves insulin signaling in mice fed a high-fat diet. Biochemical pharmacology 2012;84(11):1501-10.

109. Shah SS, Shah GB, Singh SD, et al. Effect of piperine in the regulation of obesity-induced dyslipidemia in high-fat diet rats. Indian journal of pharmacology 2011;43(3):296-9.

110. Prakash UN, Srinivasan K. Fat digestion and absorption in spice-pretreated rats. Journal of the Science of Food and Agriculture 2012;92(3):503-10.

111. Diwan V, Poudyal H, Brown L. Piperine attenuates cardiovascular, liver and metabolic changes in high carbohydrate, high fat-fed rats. Cell biochemistry and biophysics 2013;67(2):297-304.

112. Okumura $\mathrm{Y}$, Narukawa $\mathrm{M}$, Watanabe T. Adiposity suppression effect in mice due to black pepper and its main pungent component, piperine. Bioscience, biotechnology, and biochemistry 2010;74(8):1545-9.

113. Wang J, Vanegas SM, Du X, et al. Caloric restriction favorably impacts metabolic and immune/inflammatory profiles in obese mice but curcumin/piperine consumption adds no further benefit. Nutrition \& metabolism 2013;10(1):29.

114. Gregersen NT, Belza A, Jensen MG, et al. Acute effects of mustard, horseradish, black pepper and ginger on energy expenditure, appetite, ad libitum energy intake and energy balance in human subjects. The British journal of nutrition 2013;109(3):556-63.

115. Rondanelli M, Opizzi A, Perna S, et al. Improvement in insulin resistance and favourable changes in plasma inflammatory adipokines after weight loss associated with two months' consumption of a combination of bioactive food ingredients in overweight subjects. Endocrine 2013;44(2):391-401. 
116. Rondanelli M, Opizzi A, Perna S, et al. Acute effect on satiety, resting energy expenditure, respiratory quotient, glucagon-like peptide-1, free fatty acids, and glycerol following consumption of a combination of bioactive food ingredients in overweight subjects. Journal of the American College of Nutrition 2013;32(1):41-9.

117. Gilardini L, Pasqualinotto L, Di Pierro F, et al. Effects of Greenselect Phytosome(R) on weight maintenance after weight loss in obese women: a randomized placebo-controlled study. BMC complementary and alternative medicine 2016;16:233.

118. Directo D, Wong MWH, Elam ML, et al. The Effects of a Multi-Ingredient Performance Supplement Combined with Resistance Training on Exercise Volume, Muscular Strength, and Body Composition. Sports (Basel, Switzerland) 2019;7(6).

119. Zanzer YC, Plaza M, Dougkas A, et al. Black pepperbased beverage induced appetite-suppressing effects without altering postprandial glycaemia, gut and thyroid hormones or gastrointestinal well-being: a randomized crossover study in healthy subjects. Food \& function 2018;9(5):2774-86.

120. O'Connor A, Corbin KD, Nieman DC, et al. A randomized, controlled trial to assess short-term black pepper consumption on 24-hour energy expenditure and substrate utilization. Functional Foods in Health and Disease 2013;3(10):377-88.

121. Rupasinghe HP, Sekhon-Loodu S, Mantso T, et al. Phytochemicals in regulating fatty acid betaoxidation: Potential underlying mechanisms and their involvement in obesity and weight loss. Pharmacol Ther 2016;165:153-63.

122. Gupta SC, Kismali G, Aggarwal BB. Curcumin, a component of turmeric: from farm to pharmacy. Biofactors 2013;39(1):2-13.

123. Ammon HP, Wahl MA. Pharmacology of Curcuma longa. Planta Med 1991;57(1):1-7.

124. Kotha RR, Luthria DL. Curcumin: Biological, Pharmaceutical, Nutraceutical, and Analytical Aspects. Molecules 2019;24(16).
125. Nelson KM, Dahlin JL, Bisson J, et al. The Essential Medicinal Chemistry of Curcumin. J Med Chem $2017 ; 60(5): 1620-37$.

126. Kotra VSR, Satyabanta L, Goswami TK. A critical review of analytical methods for determination of curcuminoids in turmeric. I Food Sci Technol 2019;56(12):5153-66

127. Wang S, Moustaid-Moussa N, Chen L, et al. Novel insights of dietary polyphenols and obesity. J Nutr Biochem 2014;25(1):1-18.

128. Fleenor BS, Carlini NA, Campbell MS. Curcumin and arterial function in health and disease: impact on oxidative stress and inflammation. Curr Opin Clin Nutr Metab Care 2019;22(6):459-64.

129. Ding L, Li J, Song B, et al. Curcumin rescues high fat diet-induced obesity and insulin sensitivity in mice through regulating SREBP pathway. Toxicol Appl Pharmacol 2016;304:99-109.

130. Shao W, Yu Z, Chiang Y, et al. Curcumin prevents high fat diet induced insulin resistance and obesity via attenuating lipogenesis in liver and inflammatory pathway in adipocytes. PLoS One 2012;7(1):9.

131. Wright LE, Brandon AE, Hoy AJ, et al. Amelioration of lipid-induced insulin resistance in rat skeletal muscle by overexpression of Pgc-1beta involves reductions in long-chain acyl-CoA levels and oxidative stress. Diabetologia 2011;54(6):1417-26

132. Wang S, Wang X, Ye Z, et al. Curcumin promotes browning of white adipose tissue in a norepinephrine-dependent way. Biochem Biophys Res Commun 2015;466(2):247-53.

133. Ganjali S, Sahebkar A, Mahdipour E, et al. Investigation of the effects of curcumin on serum cytokines in obese individuals: a randomized controlled trial. ScientificWorldJournal 2014;11(898361).

134. Panahi $Y$, Hosseini MS, Khalili N, et al. Effects of curcumin on serum cytokine concentrations in subjects with metabolic syndrome: A post-hoc analysis of a randomized controlled trial. Biomed Pharmacother 2016;82:578-82. 
135. Saraf-Bank S, Ahmadi A, Paknahad Z, et al. Effects of curcumin supplementation on markers of inflammation and oxidative stress among healthy overweight and obese girl adolescents: A randomized placebo-controlled clinical trial. Phytother Res 2019;33(8):2015-22.
136. Kahkhaie KR, Mirhosseini A, Aliabadi A, et al. Curcumin: a modulator of inflammatory signaling pathways in the immune system. Inflammopharmacology 2019;27(5):885-900. 